\title{
Impacto del turismo rural en la economía local de la comuna Cantagallo del cantón Jipijapa
}

\section{Impact of rural tourism in the local economy of the Cantagallo community of Jipijapa county}

\author{
José Franco Yoza \\ jose.franco@unesum.edu.ec \\ Carlos Zea Barahona \\ carlos.zea@unesum.edu.ec \\ Hernán Delgado Solís \\ hernan.delgado@unesum.edu.ec \\ Sumba BustamanteYadira \\ Laura Muñiz Jaime \\ Yuly Quito Rodríguez
}

Recibido: 9/1/2019; Aceptado: 11/1/2019

\section{RESUMEN}

El presente trabajo surge de una investigación donde se refleja un estudio relacionado al impacto del turismo rural de la comuna Cantagallo del cantón Jipijapa. Partiendo de este enfoque se evidencia poca cultura turística, deficiente explotación, escasa promoción de los lugares turísticos de la zona y deficiente apoyo de las autoridades locales. Por esta razón es importante que la comunidad genere su propia economía a partir de la producción de servicios turísticos, para obtener ingresos, disminuir la pobreza y el índice de migración. La metodología fue de campo y exploratoria, los métodos deductivo-inductivo, estadístico y analítico, así como las técnicas de la observación, encuesta y entrevista. Los resultados son relevantes, porque sus habitantes saben lo que es turismo rural, pero desconocen ciertas actividades turísticas que se debe realizar. Desde esta perspectiva se presentó una propuesta de difusión y promoción de los atractivos turísticos para fomentar el desarrollo de este sector.

\footnotetext{
${ }^{1}$ Universidad Estatal del Sur de Manabí. Ecuador 2

Universidad Estatal del Sur de Manabí. Ecuador 3

Universidad Estatal del Sur de Manabí. Ecuador 4

Universidad Estatal del Sur de Manabí. Ecuador 5

Universidad Estatal del Sur de Manabí. Ecuador 6

Universidad Estatal del Sur de Manabí. Ecuador
}

Revista científica Ciencia y Tecnología Vol 20 No 25 págs. 39-45_

http://cienciaytecnologia.uteg.edu.ec 


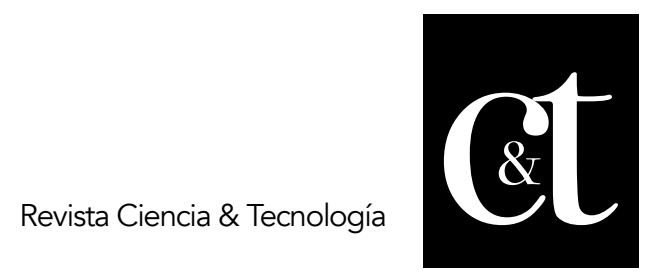

No. 25, 31 de enero de 2020

ISSN impreso: 1390 - 6321

ISSN online: 2661 - 6734

métodos deductivo-inductivo, estadístico y analítico, así como las técnicas de la observación, encuesta y entrevista. Los resultados son relevantes, porque sus habitantes saben lo que es turismo rural, pero desconocen ciertas actividades turísticas que se debe realizar. Desde esta perspectiva se presentó una propuesta de difusión y promoción de los atractivos turísticos para fomentar el desarrollo de este sector.

Palabras clave: Desarrollo local, feriados, ingresos familiares, economía turística, promoción

\begin{abstract}
The present work arises from a research that reflects a study related to the impact of rural tourism in the Cantagallo commune of the Jipijapa canton. From this perspective, information was collected on what has happened in this sector, in correlation with the participation of the inhabitants of the community who are closely linked to rural tourism, based on the use of natural and cultural resources. Who owns the area Based on this approach, there is evidence of the lack of a tourist culture on the part of its members, poor tourist exploitation, little promotion of the existing tourist sites in the area, and little support from local authorities. For this reason, it is important for the community to generate its own economy based on the production of tourism services, which will allow them to obtain income and reduce poverty and the migration rate. The methodology used was field and exploratory, and the deductive-inductive, statistical and analytical methods and techniques such as observation, survey and interview. The results obtained in this research process are relevant, because its inhabitants know what rural tourism is, but they do not know certain tourist activities that must be carried out. Here is the importance of applying different organizational formulas related to tourism, contributing greatly to rural development processes. In this sense, a proposal of diffusion and promotion of tourist attractions was presented to promote the development of this rural sector.
\end{abstract}

Keywords: Local development, holidays, family income, tourism economy, promotion

\title{
Introducción
}

El turismo rural en la actualidad es el crecimiento y desarrollo de muchas comunidades a nivel nacional e internacional, la comuna Cantagallo es un lugar que presenta muchas bondades turísticas, pero no se lo está aprovechando de la mejor manera debido a muchas razones propias de la comunidad: como el desconocimiento en temas turísticos, innovación, diseños, emprendimiento y aprovechamientos de recursos naturales.

La comuna Cantagallo es un lugar que cuenta con diversos lugares de atracción turística que utilizándolos de forma responsables podrán fomentar al desarrollo de la comuna y calidad de vida de sus habitantes. 


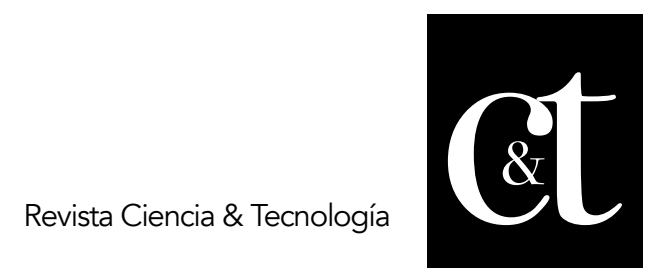

No. 25, 31 de enero de 2020

ISSN impreso: 1390 - 6321

ISSN online: 2661 - 6734

\section{Desarrollo}

Origen del turismo rural

Según (Fourneau F., 1998). Entender el origen del turismo rural (TR) implica desentramar el propósito con el cual surgió: "La yuxtaposición de las dos palabras turismo y rural puede parecer bastante contradictoria: la primera da la idea de modernidad y la segunda de tradición".

Para Combariza González (2012), otro factor que determina el nacimiento del TR, es la crisis del turismo de masas o tradicional, que origina una nueva demanda por productos innovadores que le ofrezcan al turista nuevas experiencias de ocio y de diversión.

Cabeza (2000) afirma que "la industria turística inmersa en pleno segundo boom turístico iniciado en 1990, está cambiando. El turismo de masas está en crisis, el marco de referencia se está modificando y las prácticas vinculadas al mismo deberán ser objeto de una rápida transformación, dando paso a nuevas tecnologías más productivas y eficientes. Existen indicios que apuntan hacia una "Nueva Era del Turismo" en la que imperan la calidad, la flexibilidad, la sensibilización hacia aspectos medioambientales, la individualización, la innovación y finalmente la integración vertical, horizontal y diagonal".

\section{Turismo rural y desarrollo económico}

Otra es la consideración del TR como las actividades que realizan las personas durante sus viajes y estancias en espacios rurales, con una permanencia mínima de una noche, con motivo de disfrutar de los atractivos de lo rural y de las posibilidades que ofrecen estos espacios para la satisfacción de necesidades más específicas. Las manifestaciones más comunes de TR son: el agroturismo, ecoturismo, turismo deportivo, turismo cultural, turismo de interior. (J., 2000).

Según Agrilar Criado, Merino Baena, \& Migens Fernández (2003), el turismo rural constituye uno los elementos centrales de las políticas de desarrollo y, lo que resulta aún más importante, es que la cultura local se convierte en un componente fundamental de dicha oferta turística. Una cultura que en los numerosos textos oficiales sobre desarrollo local es concebida como un recurso social y como tal puede actuar como motor de dinamización económica.

Para Flores Amador \& Zizumbo Villareal (2003), adquiere importancia el turismo rural, el cual es una alternativa de desarrollo en los ámbitos rurales, que cuenta con la participación de la comunidad local, así también, constituye una eficaz opción de progreso, aprovechando el territorio y uso racional de su riqueza patrimonial y cultural, así como, de la prestación de servicios, a través de actividades complementarias, protegiendo y conservando sus recursos naturales, desarrollando con ello, una diversificación en las acciones del campo, creando una nueva estrategia de supervivencia, posibilitando el desarrollo de lascomunidades.

El turismo rural comunitario representa una etapa avanzada del ecoturismo. El concepto de turismo rural o comunitario tiene sus raíces en Europa y describe la modalidad de alquilar una o más habitaciones de la casa del propietario a precios módicos, para propiciar la convivencia con las familias y sus costumbres (comida, forma de vivir, etc.), sin enfocar específicamente las prácticas agrícolas. (Ibídem). 


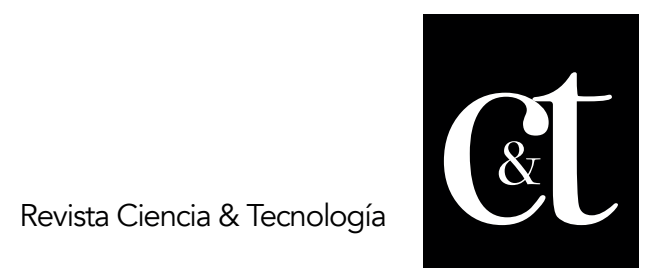

No. 25, 31 de enero de 2020

ISSN impreso: 1390 - 6321

ISSN online: 2661 - 6734

Bardón Fernández (1987) define a la oferta turística rural como el conjunto de alojamientos, instalaciones, estructuras de ocio y recursos naturales y arquitectónicos existentes en zonas de economía predominantemente agrícola.

Esta definición, presenta el inconveniente de centrarse sólo en la oferta rural, por lo que queda un tanto incompleta, sin embargo, ilustra muy bien lo que debe ser la oferta rural.

El turismo rural y el aprovechamiento de los recursos naturales

El turismo rural, para Fuentes García (1995), es aquella actividad turística realizada en el espacio rural, compuesta por una oferta integrada de ocio, dirigida a una demanda cuya motivación es el contacto con el entorno autóctono y que tenga una relación con la sociedad local.

El turismo comunitario en términos socioeconómicos, complementa y diversifica los ingresos de las familias campesinas, combate el aislamiento económico, desarrolla capacidad empresarial, contribuye a frenar la migración rural, permite valorar y recuperar la cultura local y suscita el desarrollo de infraestructura en zonas rurales. (Flores Amador \& Zizumbo Villareal, 2003). En términos ambientales, el turismo rural comunitario desarrolla capacidades en las comunidades para brindar servicios ambientales, al convertirse en un incentivo para la conservación como en el caso de reservas privadas que conforman corredores biológicos, o estimulando acciones de protección y prácticas productivas sostenibles. (Ibídem).

De acuerdo a Erique Piedra (2014), "el turismo en los espacios rurales, se ha convertido en un verdadero motor de desarrollo, aunque es clara la inexistencia de un acuerdo generalizado sobre el modelo adecuado de gestión. Un desarrollo integral de carácter endógeno en el medio rural se basa en la explotación de los recursos del medio natural, de los recursos demográficos y de aquellos otros que se han ido forjando durante la historia y del resto de los recursos que puedan tener un valor económico".

En el Ecuador, el Ministerio de Información y Turismo fue creado el 10 de agosto de 1992, a inicios del gobierno de Sixto Durán Ballén. Este visualizó al turismo como una actividad fundamental para el desarrollo económico y social de los pueblos. Frente al crecimiento del sector turístico, en junio de 1994, se tomó la decisión de separar al turismo de la información, para que se dedicara exclusivamente a impulsar y fortalecer esta actividad. (Urgiles Andrade, 2011).

Cantagallo es una comunidad que pertenece a la parroquia Puerto Cayo del cantón Jipijapa. Aquella comunidad cuenta con atractivos turísticos tales como el bosque seco, gastronomía, tolas, fincas orgánicas, piletas de agua, barro y su principal eje de atracción es el de los monos aulladores (nativos del mismo lugar, presentes los 365 días del año).

\section{Metodología}

Entre los principales métodos que permitieron el desarrollo del presente estudio están el método deductivo, inductivo, hipotético-deductivo, estadístico y analítico apoyado de las técnicas como la observación, la encuesta y la entrevista. Se contó con una muestra de la población de Cantagallo la misma que sumó un total de 234 habitantes, de los cuales se obtuvo el consentimiento informado. 


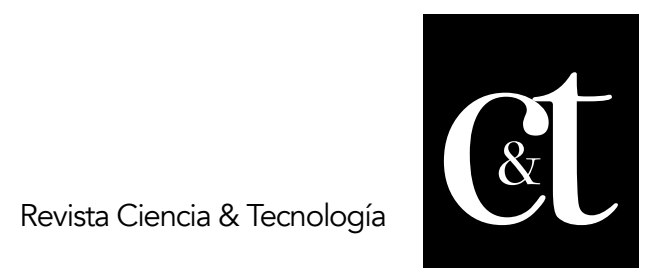

No. 25, 31 de enero de 2020

ISSN impreso: 1390 - 6321

ISSN online: 2661 - 6734

\section{Resultados}

Se pudo identificar que entre las principales actividades a las que se dedican los habitantes de la comuna Cantagallo están las siguientes:

\section{Tabla 1. ¿A qué actividad se dedica usted?}

\begin{tabular}{lll}
\hline Alternativa & $\mathrm{N}^{\circ}$ & Porcentaje \\
\hline Agricultor & 152 & $65 \%$ \\
Ama de casa & 35 & $15 \%$ \\
Comerciante & 23 & $10 \%$ \\
Empleado publico & 5 & $2 \%$ \\
Negocio propio & 17 & $7 \%$ \\
Otro & 2 & $1 \%$ \\
Total & 234 & $100 \%$ \\
\hline
\end{tabular}

Fuente: Elaboración propia

La principal actividad es la agricultura, el $65 \%$ de los encuestados realizan actividades en sus parcelas. Del mismo al ser consultado los encuestados sobre el conocimiento que tienen sobre lo que es el turismo rural, el $70 \%$ respondió que sí conocen lo que es esta actividad, según se lo muestra en la tabla 2:

Tabla 2. ¿Conoce usted sobre el turismo rural?

\begin{tabular}{lll}
\hline Alternativa & $\mathrm{N}^{\circ}$ & Porcentaje \\
\hline $\mathrm{Si}$ & 164 & $70 \%$ \\
$\mathrm{No}$ & 70 & $30 \%$ \\
Total & 234 & $100 \%$ \\
\hline
\end{tabular}

Fuente: Elaboración propia

Cuando se les pregunto si en la comunidad existen atractivos turísticos, los consultados manifestaron en el $70 \%$ que sí existen, pero que estos no están siendo explotados debidamente por los habitantes de la comunidad, ya que evidencia que semanalmente una a dos veces llegan personas a deleitar de la naturaleza que existe en el medio, así como a consumir los productos agrícolas que se producen en la comunidad, además se pueden observar que en épocas de feriados llegan más los turistas, entre los cuales están los nacionales en su mayoría y los extranjeros:

Tabla 3. ¿Cada que tiempo llega un turista a la comunidad?

\begin{tabular}{lll} 
Alternativa & $\mathrm{N}^{\circ}$ & Porcentaje \\
\hline Todos los dias & 0 & $0 \%$ \\
Dos veces por & 47 & $20 \%$ \\
semana & & \\
$\begin{array}{l}\text { De repente } \\
\text { Solo en feriados }\end{array}$ & 47 & $20 \%$ \\
Total & 140 & $60 \%$ \\
\hline
\end{tabular}

Fuente: Elaboración propia 


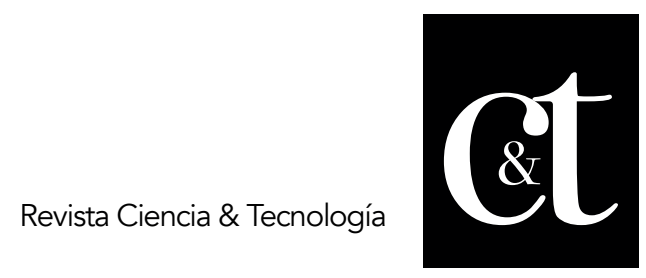

No. 25, 31 de enero de 2020

ISSN impreso: 1390 - 6321

ISSN online: 2661 - 6734

Del mismo modo al preguntarle a los encuestados sobre la importancia que los habitantes están asignado al desarrollo del turismo rural en la comunidad, manifestaron el $70 \%$ que no se le da mayor importancia, esto se debe a que existen factores que limitan el desarrollo de esta actividad y existe poco fomento a crear inversiones para impulsar el turismo rural, en cambio al consultar si el turismo rural aportará al desarrollo económico de la comunidad, el 70\% expreso que si, por lo tanto es muy importante impulsar estas actividades ya que esto significan nuevos ingresos, generación de fuentes de empleo que tanto necesita esta localidad, esto se lo muestra en la siguiente tabla:

Tabla 4. ¿Cree usted que el turismo rural aportará al desarrollo económico en la comunidad de Cantagallo?

\begin{tabular}{lll}
\hline Alternativa & $\mathrm{N}^{\circ}$ & Porcentaje \\
\hline $\mathrm{Si}$ & 164 & $70 \%$ \\
$\mathrm{No}$ & 70 & $30 \%$ \\
Total & 234 & $100 \%$ \\
\hline
\end{tabular}

Fuente: Elaboración propia.

En relación a si las autoridades del cantón y de la parroquia se preocupan por promocionar los recursos naturales que existen en la comunidad, los encuestados expresaron que no existe ninguna actividad que promocione los recursos naturales de Cantagallo, que a pesar de estar cerca de la cabecera parroquial cuando existen feriados o en épocas de carnaval no se los incluye en susplanificaciones.

Tabla 5. ¿Se promocionan los lugares turísticos que existen en la comunidad?

\begin{tabular}{lll} 
Alternativa & $N^{\circ}$ & Porcentaje \\
\hline Si & 70 & $30 \%$ \\
No & 164 & $70 \%$ \\
Total & 234 & $100 \%$ \\
\hline
\end{tabular}

Fuente: Elaboración propia.

Del mismo modo se les pregunto si desean ser capacitados en temas relacionados con el turismo rural, sobre sus ventajas y desventajas y la oportunidad que esta actividad genera en cuanto a recibir nuevos ingresos, los encuestados indicaron en el $98 \%$ que si desean recibir estas capacitaciones. Se muestra un interés muy significativo por la necesidad de buscar nuevas alternativas para mejorar en los aspectos socioeconómicos personales y de la comunidad.

\section{Discusión}

En la comunidad de Cantagallo del cantón Jipijapa, si existen atractivos turísticos relacionados con el turismo rural, esto es un factor muy importante para el desarrollo económico de los habitantes que se encuentran en su entorno, de acuerdo a Maldonado (2006):

El turismo es un fenómeno económico en diversas esferas de la economía, dinamizando las políticas de fenómeno de negocios, estimulando la demanda de bienes y servicios de una alta gama de actividades locales, lo cual significa generación 


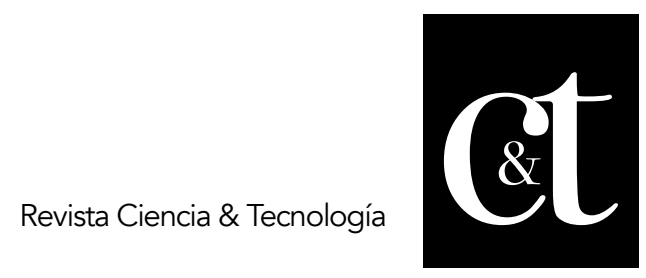

No. 25, 31 de enero de 2020

ISSN impreso: 1390 - 6321

ISSN online: 2661 - 6734

de la demanda, inflación de precios, especulación de todo tipo y fuga de los beneficios, vía compras en el extranjero o expatriación de beneficios.

Igualmente, para (Urgilés Andrade, 2011). El turismo influye económicamente en el desarrollo de las comunidades a nivel mundial, en el llamado turismo comunitario, en el que todas las familias de un lugar determinado, por medio de la actividad turística, obtienen un beneficio para su comunidad, mejorando así su nivel de vida.

Según (Fontana Coto, 2012). La potencialidad de un territorio se localiza en un entorno específico, con sujetos que por lo general están marcados por identidad del lugar y quienes son los que dinamizan ese territorio, con sus propias capacidades en armonía con el potencial natural característico que hace único a dicho territorio, ya que no pasa lo mismo en un territorio " $x$ " $y$ en un territorio " $y$ ", porque en ambos intervienen particularidades propias, aunado a los habitantes que son el centro de esas dinámicas, más las características ambientales y culturales, es lo que marca el desarrollo de ese territorio o localidad y la hace particular.

\section{Conclusiones}

El Turismo Rural en la actualidad es un factor esencial en el desarrollo de las comunidades, en la comuna Cantagallo del cantón Jipijapa existe lugares turísticos no aprovechados como sus bosques, que son visitados con más frecuencia por personas nacionales y extranjeras, generando mejoras a la comuna en el aspecto socioeconómico.

Los habitantes de la comuna Cantagallo del cantón Jipijapa se beneficiarán con el desarrollo del turismo rural ya que este permite la atracción de turistas que apreciarán cada uno de sus lugares, produciendo no solo beneficios económicos sino incentivando a la planta turística a cambiar sus actividades no sostenibles por acciones que generen rentabilidad y beneficios para todos.

\section{Referencias bibliográficas}

Agrilar Criado, E., Merino Baena, D., \& Migens Fernández, M. (2003). Cultura, Políticas de Desarrollo y Turismo Rural en el Ámbito de la Globalización. España: Universidad de Sevilla.

Bardón Fernández, E. (1987). El Turismo Rural en España. Estudios Turísticos. N94 1987, págs. 63-76.

Cabeza M. (2000). La industria turística busca nuevos horizontes. Economía, XXV, 16 (2000), pp. 33-44.

Combariza González, J. (2012). El turismo rural como estrategia de desarrollo sostenible: caso municipio de La Mesa (Cundinamarca). Bogotá: Universidad Nacional de Colombia.

Erique Piedra, M. C. (2014). El Turismo Rural Comunitario y la Influencia en el Desarrollo de Parroquia Quinara, Cantón Loja. Loja: Universidad Nacional de Loja. 